\title{
SCREENING OF A POSITRON IN AN INHOMOGENEOUS ELECTRON GAS
}

\author{
H. STACHOWIAK \\ W. Trzebiatowski Institute for Low Temperature and Structure Research \\ Polish Academy of Sciences \\ P.O. Box 937, 50-950 Wrocław 2, Poland
}

\begin{abstract}
Calculations of the local annihilation rate of positrons in inhomogeneous media have been recently performed. However, the problem was solved only for the positron at the center of the inhomogeneity. This allowed to benefit of the spherical symmetry of the problem. Since the positron is usually an itinerant particle, it is necessary in practical applications to perform computations for every possible coordinates of this particle. In the present work local annihilation rates were obtained for noncentral coordinates of the positron.
\end{abstract}

PACS numbers: $78.70 . \mathrm{Bj}, 71.60 .+\mathrm{z}$

\section{Introduction}

The approach to electron-positron interaction (EPI) was developed by Kallio, Pietiläinen and Lantto [1-3] and labelled HNC (hypernetted-chain) benefits of the achievements of the theory of liquids. In the form proposed by Gondzik and Stachowiak [4], it proved to be particularly efficient and leading to reasonable results (cf. also [5-8]) as well for positron lifetime, EPI correlation energy and partial annihilation rates. The partial annihilation rates, however, are obtained after development of the theory by perturbing the Jastrow-type trial function of HNC in order to allow for momentum dependence in electron-positron scattering $[5,6]$. The simplicity of the HNC approach makes it a privileged one while investigating EPI in an inhomogeneous medium, e.g. a metal lattice.

Indeed, EPI in inhomogeneous media is still an unsolved theoretical problem. In spite of quite a few attempts to understand the mechanism of screening the positron in a metal, the subject remains very controversial. The classical approach is the independent particle model (IPM) still used with success in most experimental Fermi surface studies. If EPI enhances the contact density of all electronic states on the positron by the same factor, IPM is a better approximation than would follow from its name, at least as concerns the angular correlation experiment. 
Many theoretical works used the local density approximation (LDA) to EPI [9-15]. Works using the constant potential approximation should be mentioned also [16-18], as well as their generalizations [19]. The common defect of all these approaches is that self-consistency of EPI is not controlled, i.e. it is not known whether the potential used in the formalism leads to exact screening of the positron, in other words is the total screening charge equal to one electronic charge.

The HNC approach in the form proposed by Gondzik and Stachowiak [4] has been used for studying EPI in inhomogeneous media [7], including metal lattices (for the case of a positron in lithium) [20]. This allowed to check how justified are the approximations applied usually in positron physics, namely IPM and LDA. It was found that in many cases the local annihilation rate is roughly proportional to the local electron density (in the absence of the positron), so, as concerns angular correlation, IPM seems to be not so bad approximation, as known for a long time by experimentalists ( $w c$ leave here aside the problem of momentum dependent enhancement which cannot be treated in pure HNC). However, technical reasons prevented the authors of Refs. [7] and [20] from treating cases where the medium surrounding the positron is direction dependent, so one single one-dimensional function cannot describe the screening.

In the present work a formalism is developed in which the local annihilation rates are computed for a positron inside a spherical inhomogeneity in an electron liquid. Since the positron is more or less delocalized, all its positions with regard to the inhomogeneity must be taken into account, especially those where the positron coordinates do not coincide with the center of the inhomogeneity.

\section{Basic assumptions}

We derived in [20] the equation for the enhancement amplitude $w$ in a metal lattice

$$
\chi\left[-\nabla^{2}+W\left(r_{\mathrm{p}}, r\right)\right] w-2 \nabla \chi \nabla w=0 .
$$

$\chi(r)$ - the density amplitude of conduction electrons in the absence of the positron - is a known function. We will assume that it has spherical symmetry. $r_{\mathrm{p}}$ - the positron coordinate - differs from zero. The derivatives are taken with respect to $r$.

Equation (1) is obtained in the following way: $\chi(r)$ obeys the Euler-Lagrange type equation

$$
\left[-\frac{1}{2} \nabla^{2}+V^{0}(\boldsymbol{r})\right] \chi(\boldsymbol{r})=\eta \chi(\boldsymbol{r})
$$

where $V^{0}(r)$ is the self-consistent lattice potential and $\eta$ is the Lagrange multiplier. In presence of the positron at $r_{\mathrm{p}}$ the density amplitude takes the form $\zeta\left(r_{\mathrm{p}}, r\right)$ and obeys the equation

$$
\left[-\frac{1}{2} \nabla^{2}+V^{0}(r)+\frac{1}{2} W\left(r_{\mathrm{p}}, r\right)\right] \zeta\left(r_{\mathrm{p}}, r\right)=\eta \zeta\left(r_{\mathrm{p}}, r\right)
$$

where $W\left(r_{\mathrm{p}}, r\right)$ is the screened electron-positron potential. Substituting 


$$
\zeta\left(r_{\mathrm{p}}, r\right)=w\left(r_{\mathrm{p}}, r\right) \chi(r)
$$

into Eq. (3) one gets Eq. (1).

The screened potential of electron-positron interaction $W$ consists of three parts

$$
W\left(r_{\mathrm{p}}, r\right)=-\frac{1}{\left|\boldsymbol{r}-r_{\mathrm{p}}\right|}+W_{\mathrm{p}}\left(r_{\mathrm{p}}, \boldsymbol{r}\right)+W_{\mathrm{xc}}\left(r_{\mathrm{p}}, r\right),
$$

where the Coulomb potential of the screening cloud is expressed as

$$
W_{\mathrm{p}}\left(r_{\mathrm{p}}, r\right)=\cdot \int \mathrm{d} r^{\prime} \frac{w^{2}\left(r_{\mathrm{p}}, r^{\prime}\right)-1}{\left|r-r^{\prime}\right|} \chi^{2}\left(r^{\prime}\right)
$$

and the exchange-correlation contribution is assumed as

$$
W_{\mathrm{xc}}\left(r_{\mathrm{p}}, \boldsymbol{r}\right)=V_{\mathrm{HL}}\left\{w^{2}\left(\boldsymbol{r}_{\mathrm{p}}, \boldsymbol{r}\right) \chi^{2}(r)\right\}-V_{\mathrm{HL}}\left\{\chi^{2}(r)\right\} \text {, }
$$

where $V_{\mathrm{HL}}$ has the form proposed by Hedin and Lundqvist [2I].

We intend to solve in this work the problem of positron screening in an inhomogeneous electron gas in which the isolated spherical inhomogeneity concerns the electron density in absence of the positron and is expressed as

$$
\rho(r)=\chi^{2}(r)=\rho_{0}\left\{1+\sigma \exp \left[-\left(\frac{r}{s_{0}}\right)^{2}\right]\right\} .
$$

$\sigma(\sigma>-1)$ and $s_{0}$ are parameters of obvious physical meaning: $\sigma$ represents the amplitude and $s_{0}$ the size of the inhomogeneity. $\rho_{0}$ is the density of the electron gas. In the present work it corresponds to the density of conduction electrons in lithium $\left(r_{\mathrm{s}}=3.248\right)$.

A similar model has already been investigated [20] for the positron in the center of the inhomogeneity. Here we will consider the problem of a non-central positron.

\section{Equation for the enhancement amplitude}

The enhancement amplitude can be written as an expansion in Legendre polynomials

$$
w\left(r_{\mathrm{p}}, r\right)=A\left(r_{\mathrm{p}}\right) \mathrm{e}^{-\alpha s}+\tau\left(r_{\mathrm{p}}, r\right)
$$

where

$$
\tau\left(r_{\mathrm{p}}, r\right)=\sum_{n=0}^{\infty} \varphi_{n}\left(r_{\mathrm{p}}, r\right) P_{n}(\cos \vartheta)
$$

vanishes at $r=r_{\mathrm{p}}$ and is devoid of the cusp occurring in $w$ at the positron

$$
s=\left|r-r_{\mathrm{p}}\right|=\left(r^{2}+r_{\mathrm{p}}^{2}-2 r r_{\mathrm{p}} \cos \vartheta\right)^{1 / 2} .
$$

From the assumption $\tau\left(r_{\mathrm{p}}, r_{\mathrm{p}}\right)=0$ it follows that $\alpha=1 / 2$.

The value of $A\left(r_{\mathrm{p}}\right)$ gives information about the local annihilation rate. $\vartheta$ is the angle between $r$ and $r_{\mathrm{p}}$. The smoothness of $\tau$ allows to limit ourselves, at least in this work, to two terms in the expansion (10).

With two components left in the expansion (10) and a nodeless unperturbed density amplitude $\chi(r)$ Eq. (1) takes a more specific form

$$
-\varphi_{0}^{\prime \prime}-2\left(\frac{1}{r}+\frac{\chi^{\prime}}{\chi}\right) \varphi_{0}^{\prime}+W\left(r_{\mathrm{p}}, r\right) \varphi_{0}
$$




$$
\begin{aligned}
& +\left[-\varphi_{1}^{\prime \prime}-2\left(\frac{1}{r}+\frac{\chi^{\prime}}{\chi}\right) \varphi_{1}^{\prime}+\left(W\left(r_{\mathrm{p}}, r\right)+\frac{2}{r^{2}}\right) \varphi_{1}\right] \cos \vartheta \\
& +\left[-\alpha^{2}+\frac{2 \alpha}{s}\left(1+\frac{\chi^{\prime}}{\chi} r\right)-\frac{2 \alpha}{s} \frac{\chi^{\prime}}{\chi} r_{\mathrm{p}} \cos \vartheta+W\left(r_{\mathrm{p}}, r\right)\right] A \mathrm{e}^{-\alpha s}=0
\end{aligned}
$$

We solve Eq. (12) as usual by the way of successive linearizations, substituting for $\varphi_{0}, \varphi_{1}$ and $A$ the forms

$$
\varphi_{0}=\varphi_{0}^{0}+\delta \varphi_{0}, \quad \varphi_{1}=\varphi_{1}^{0}+\delta \varphi_{1}, \quad A=A_{0}+\delta A,
$$

where $\varphi_{0}^{0}, \varphi_{1}^{0}$ and $A_{0}$ are assumed to be known and $\delta \varphi_{0}, \delta \varphi_{1}$ and $\delta A$ to be small, so terms nonlinear in these quantities can be omitted. Usually 5 linearizations are sufficient to obtain a satisfactory solution.

In this way we get from (12) a linearized equation for $\delta \varphi_{i}$ and $\delta A$.

By expressing this equation in terms of spherical harmonics we could obtain from it an infinite series of one-dimensional equations describing the angular behaviour of the solution. However, since we limit ourselves to two terms in the expansion, the same must be done while solving this equation. Namely, we must expand both sides of it into spherical harmonics, limiting ourselves to the first two terms.

In this way we get finally the equations

$$
\begin{aligned}
& -\delta \varphi_{0}^{\prime \prime}-2\left(\frac{1}{r}+\frac{\chi^{\prime}}{\chi}\right) \delta \varphi_{0}^{\prime}+\left(\widetilde{\mathcal{W}}_{1}+\zeta_{00}\right) \delta \varphi_{0} \\
& +\left(\widetilde{\mathcal{W}}_{2}+\zeta_{01}\right) \delta \varphi_{1}+\left(\mathcal{B}_{1}+\widetilde{\mathcal{W}}_{4}+\zeta_{0 A}+\Sigma^{0}\right) \delta A \\
& \quad+8 \pi \int_{0}^{\infty} r^{\prime 2} \mathrm{~d} r^{\prime} \chi^{2}\left(r^{\prime}\right)\left[\sigma_{0}^{0} \delta \varphi_{0}\left(r^{\prime}\right)+\sigma_{1}^{0} \delta \varphi_{1}\left(r^{\prime}\right)\right]=-F_{0}, \\
& -\delta \varphi_{1}^{\prime \prime}-2\left(\frac{1}{r}+\frac{\chi^{\prime}}{\chi}\right) \delta \varphi_{1}^{\prime}+\left(3 \widetilde{\mathcal{W}}_{2}+\zeta_{10}\right) \delta \varphi_{0} \\
& +\left(\frac{2}{r^{2}}+3 \widetilde{\mathcal{W}_{3}}+\zeta_{11}\right) \delta \varphi_{1}+\left(3 \mathcal{B}_{2}+3 \widetilde{\mathcal{W}}_{5}+\zeta_{1 A}+\Sigma^{1}\right) \delta A \\
& +8 \pi \int_{0}^{\infty} r^{\prime 2} \mathrm{~d} r^{\prime} \chi^{2}\left(r^{\prime}\right)\left[\sigma_{0}^{1} \delta \varphi_{0}\left(r^{\prime}\right)+\sigma_{1}^{1} \delta \varphi_{1}\left(r^{\prime}\right)\right]=-F_{1} .
\end{aligned}
$$

These equations form the basis for the solution of the problem. The unknown functions $\delta \varphi_{0}, \delta \varphi_{1}$ and also $\delta A$ occur only explicitly. The other quantities are well determined, but more than eighty functions needed to define them will be omitted in this article. Let us mention only that $\widetilde{\mathcal{W}}_{i}$ are computed from the starting value of the potential $W$, the correction $\delta W$ to the potential leads to the terms $\zeta_{i j}, \Sigma^{i}$ and $\sigma_{i}^{j}, \mathcal{B}_{i}$ are due to the remaining terms being the coefficients to $A$ in Eq. (12) and $F_{i}$ vanish if $\varphi_{0}^{0}, \varphi_{1}^{0}$ and $A_{0}$ are the solution of Eq. (12). 


\section{Computations}

It is assumed that a sufficiently good description of the functions $\delta \varphi_{k}(r)$ is given by their values at a discrete set of points $r_{i}, i=1, \ldots, N$. Under this assumption Eqs. (14), (15) which in fact form a set of two integro-differential equations can be written in the form of a set of linear algebraic equations for the unknown quantities $\delta \varphi_{k}\left(r_{i}\right)$ and $\delta A$.

The boundary conditions are obvious

$\varphi_{0}^{\prime}\left(r_{\mathrm{p}}, 0\right)=0, \quad \varphi_{0}\left(r_{\mathrm{p}}, \infty\right)=1, \quad \varphi_{1}\left(r_{\mathrm{p}}, 0\right)=0, \quad \varphi_{1}\left(r_{\mathrm{p}}, \infty\right)=0$.

The quantity $\delta A$ rises the necessity of introducing an additional equation giving the criterion for the choice of $A$ in the formula (9). In practice here is the major source of the uncertainties occurring in the solution.

In our calculations we used as additional condition the equation

$\varphi_{0}\left(r_{\mathrm{p}}, r_{\mathrm{p}}\right)+\varphi_{1}\left(r_{\mathrm{p}}, r_{\mathrm{p}}\right)=v\left(r_{\mathrm{p}}\right)$

where $v\left(r_{\mathrm{p}}\right)$ was chosen according to some suitable interpolation scheme. The additional condition should be

$$
\tau\left(r_{\mathrm{p}}, r_{\mathrm{p}}\right)=0 \text {. }
$$

However, describing $\tau$ with two spherical harmonics only, we are unable to reproduce the zero. For this reason we ùsed in our calculations the additional condition in the form (17).

Our first attempt to solve Eqs. (14), (15) was performed for a homogeneous electron gas. Here we are in the advantageous situation that the exact solution of the problem is known. It is obtained for the positron at $r_{\mathrm{p}}=0$. Transferring the positron to a point away from the central point of the coordinate system we can compute the functions $\varphi_{k}^{j}\left(r_{\mathrm{p}}, r\right)$ of the expansions $(9),(10)$ for this solution.

The function $v\left(r_{\mathrm{p}}\right)$ is obtained by analogy to $(17)$ as

$v^{j}\left(r_{\mathrm{p}}\right)=\varphi_{0}^{j}\left(r_{\mathrm{p}}, r_{\mathrm{p}}\right)+\varphi_{1}^{j}\left(r_{\mathrm{p}}, r_{\mathrm{p}}\right)$.

Let us solve now Eqs. (14), (15) with the boundary condition (16), with $v\left(r_{\mathrm{p}}\right)$ given by Eq. (19), but assuming in Eq. (8) a value of $\sigma$ equal to 0 . We solve in this way the problem of screening the positron in a homogeneous electron gas, but using a coordinate system displaced with regard to the positron. The results for $A\left(r_{\mathrm{p}}\right)$ are given in-Table. We consider them as a test of the accuracy of our calculations. For a homogeneous electron gas $A\left(r_{\mathrm{p}}\right)$ - the enhancement amplitude for the positron at $r_{\mathrm{p}}$ - should not depend on $r_{\mathrm{p}}$. It is visible that for not very large values of $r_{p}$ the formalism developed in this work leads to quite reliable figures for the annihilation rate, provided appropriate values for $v\left(r_{\mathrm{p}}\right)$ are used in Eq. (17).

We present in the figures results obtained for $\sigma=-0.9$ and 2 , and 2 different values of $s_{0}$.

Figure 1 shows the annihilation rates obtained from our calculations for $\sigma=-0.9$ (full curve) compared to the annihilation rates following from LDA (dotted curve). The density distribution (in absence of the positron) is also shown (dashed curve) in order to compare the annihilation rate with the constant enhancement approximation which as concerns the angular correlation experiment 

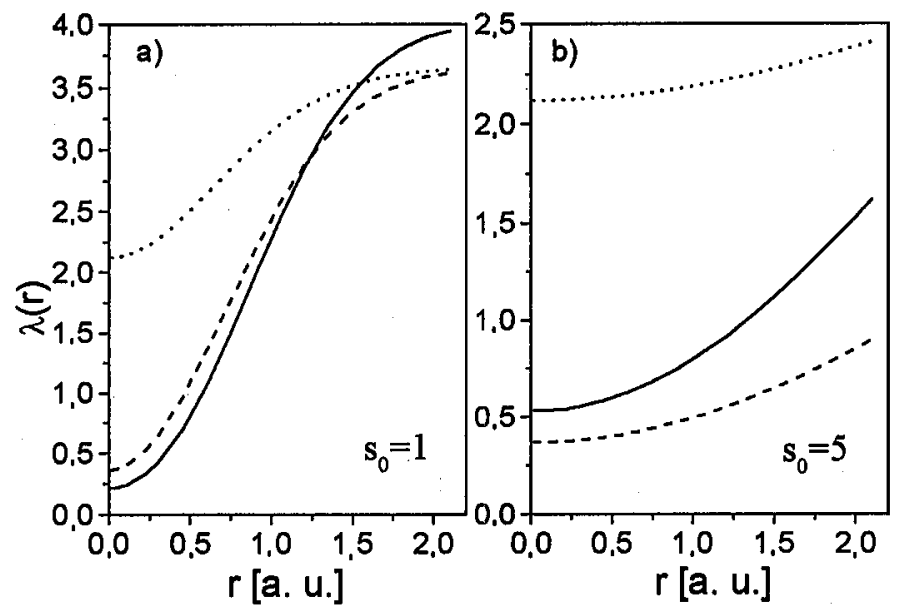

Fig. 1. Local annihilation rate $\lambda\left(r_{p}\right)$ according to this work (full curve) and according to the local density approximation (dotted curve). The dashed curve shows the electron density normalized as described in the text. $\sigma$ is equal to -0.9 and $s_{0}$ is given in the figures in atomic units.
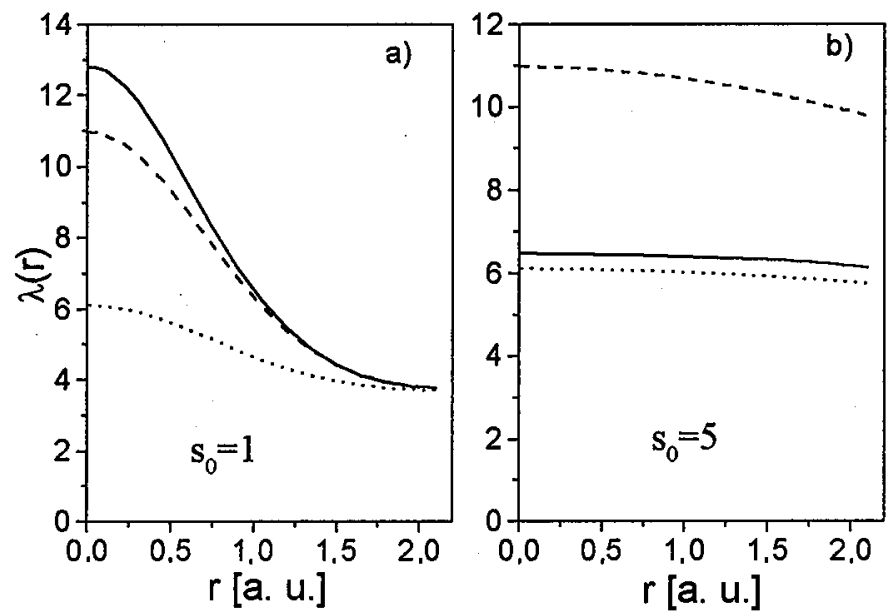

Fig. 2. The same as in Fig. 1 but for $\sigma=2$.

is equivalent to the independent-particle model. The density distribution is normalized in such a way that its value at infinity is equal to the corresponding value of the annihilation rate (for a homogeneous electron gas).

Figure 2 gives the analogous quantities for $\sigma=2$.

One can see that unless the inhomogeneity becomes very wide (large values of $s_{0}$ ), the IPM model gives a better description of the enhancement than the local density approximation. In order to get an idea about the importance of the size of the inhomogeneities let us mention that in lithium the node in the wave function of conduction electrons occurs at about 0.9 atomic units from the nucleus, while the distance between nearest neighbours is equal to $5.71 \mathrm{a} . \mathrm{u}$. 
TABLE

Dependence of $A$ as obtained from Eqs. (14), (15) for a homogeneous electron gas on the positron coordinate $r_{\mathrm{p}}\left(r_{\mathrm{s}}=3.248\right)$.

\begin{tabular}{c|c}
\hline \hline $\begin{array}{c}r_{\mathrm{p}} \\
\text { in atomic units }\end{array}$ & $A\left(r_{\mathrm{p}}\right)$ \\
\hline 0.01125 & 3.25004 \\
0.0525 & 3.25005 \\
0.1125 & 3.25006 \\
0.2250 & 3.25023 \\
0.30 & 3.25051 \\
0.45 & 3.25151 \\
0.60 & 3.25342 \\
0.75 & 3.25689 \\
0.90 & 3.26200 \\
1.05 & 3.26851 \\
1.20 & 3.27615 \\
1.35 & 3.28458 \\
1.50 & 3.29367 \\
1.65 & 3.30287 \\
1.80 & 3.31210 \\
1.95 & 3.32083 \\
2.10 & 3.32910 \\
&
\end{tabular}

\section{Conclusions}

We consider as a success of the approach that the local annihilation rate $\lambda\left(r_{\mathrm{p}}\right)$ obtained from solving Eqs. (14), (15) tends when $r_{\mathrm{p}} \rightarrow 0$ to the value $\lambda(0)$ computed for the isotropic case. This is due to the fact that the cusp term in the formula for $w(9)$ has been separated out. Otherwise a proper description of this singularity would need introducing an unrealistic number of spherical harmonics.

The results obtained in this work confirm our earlier suggestions that the local density approximation as concerns $\mathrm{e}^{+}-\mathrm{e}^{-}$interaction in an inhomogeneous electron liquid seems in most cases far from reality (as already pointed out in [7] and [20]). We would like to emphasize the role of the gradient term in Eq. (1) which replaces in some way the lattice potential. From the local density approximation it would follow that the enhancement factor increases in regions of low electron density and decreases in regions of high electron density. However, a high electron density is the result of an attractive potential which should act also on the electrons screening the positron, increasing in this way the enhancement factor, against expectations of the local density approximation. The same kind of argument applies to regions of low electron density. 
This work benefitted from grant No. 2 P03B 09912 of the Committee for Scientific Research.

\section{References}

[1] A. Kallio, P. Pietiläinen, L. Lantto, Phys. Scr. 25, 943 (1982).

[2] P. Pietiläinen, A. Kallio, Phys. Rev. B 27, 224 (1983).

[3] E. Boroński, R.M. Nieminen, Phys. Rev. B 34, 3820 (1986).

[4] J. Gondzik, H. Stachowiak, J. Phys. C 18, 5399 (1985).

[5] H. Stachowiak, Phys. Rev. B 41, 12522 (1990).

[6] H. Stachowiak, J. Lach, Phys. Rev. B 48, 9828 (1993).

[7] H. Stachowiak, Phys. Rev. B 56, 4545 (1997).

[8] E. Boroński, H. Stachowiak, Phys. Rev. B 57, 6215 (1998).

[9] J. Arponen, P. Hautojärvi, R. Nieminen, E. Pajanne, J. Phys. F 3, 2092 (1973).

[10] E. Bonderup, J.U. Andersen, D.N. Lowy, Phys. Rev. B 20, 883 (1979).

[11] B. Chakraborty, Phys. Rev. B 24, 7423 (1981).

[12] S. Daniuk, G. Kontrym-Sznajd, J. Mayers, A. Rubaszek, H. Stachowiak, P.A. Walters, R. West, in: Positron Annihilation, Eds. P.C. Jain, R.M. Singru, K.P. Gopinathan, World Scientific, Singapore 1985, pp. 43, 279; S. Daniuk, G. Kontrym-Sznajd, A. Rubaszek, H. Stachowiak, J. Mayers, P.A. Walters, R. West, J. Phys. F 17, 1365 (1987).

[13] T. Jarlborg, A.K. Singh, Phys. Rev. B 36, 4460 (1987).

[14] K.O. Jensen, J. Phys., Condens. Matter 1, 10595 (1989); S. Daniuk, M. Šob, A. Rubaszek, Phys. Rev. B 43, 2580 (1991); B. Barbiellini, P. Genoud, T. Jarlborg, J. Phys., Condens. Matter 3, 7631 (1991); M.J. Puska, J.. Phys., Condens. Matter 3, 3455 (1991).

[15] B. Barbiellini, M.J. Puska, T. Torsti, R.M. Nieminen, Phys. Rev. B 51, 7341 (1995).

[16] J.P. Carbotte, Phys. Rev. 144, 309 (1966).

[17] J.P. Carbotte, A. Salvadori, Phys. Rev. 162, 290 (1967).

[18] H. Sormann, W. Puff, in: Proc. 7th Int. Conf. on Positron Annihilation, New Delhi (India) 1985, Eds. P.C. Jain, R.M. Singru, World Scientific, Singapore 1985, p. 161.

[19] H. Sormann, Phys. Rev. B 54, 4558 (1996).

[20] H. Stachowiak, E. Boroński, G. Banach, Nukleonika 42, 237 (1997); Materiaty 29 Seminarium Anihilacji Pozytonów, Jarnottówek (Poland) 1997, Uniwersytet Opolski, Uniwersytet Wroclawski, Opole 1997, p. 89 (in Polish) and to be published.

[21] L. Hedin, B.J. Lundqvist, J. Phys. C 4, 2064 (1971). 